\title{
A modified principal component analysis-based utility theory approach for optimization of correlated responses of EDM process
}

\author{
Rina Chakravorty ${ }^{1}$, Susanta Kumar Gauri ${ }^{2 *}$, Shankar Chakraborty ${ }^{3}$ \\ ${ }^{1} S Q C \&$ OR Unit, Indian Statistical Institute, New Delhi, INDIA \\ ${ }^{2} S O C \&$ \& Unit, Indian Statistical Institute, Kolkata, INDIA \\ ${ }^{3}$ Department of Production Engineering, Jadavpur University, Kolkata, INDIA \\ *Corresponding author: e-mail: susantagauri@hotmail.com,Tel+91-033-25753350,Fax+91-033-25776042
}

\begin{abstract}
Electrical discharge machining (EDM) is one of the most extensively used non-traditional machining processes having multiple performance characteristics, some of which are usually correlated. So, ideally, use of principal component analysis (PCA)-based approaches that take into account the possible correlations between the responses are suitable for optimization of EDM process. A recently reported study reveals that PCA-based proportion of quality loss reduction (PQLR) method results in the best optimization performance among the four considered PCA-based approaches for EDM process. This paper presents a modified PCA-based utility theory (UT) approach for optimization of correlated responses. The reported experimental data on EDM processes in literature are analyzed using the modified PCA-based UT approach and PCA-based PQLR method. Comparison of the predicted performance measures at the optimal process conditions derived based on these two PCA-based approaches reveal that the modified PCA-based UT approach leads to better overall optimization performance. So, it can be the most promising approach for optimizing EDM processes.
\end{abstract}

Keywords: EDM; Correlated responses; Optimization; Principal component analysis; Proportion of quality loss reduction; Utility theory.

DOI: http://dx.doi.org/10.4314/ijest.v4i2.3

\section{Introduction}

Electrical discharge machining (EDM) has become one of the most extensively used non-traditional material removal processes. Researchers have successfully applied EDM process to machine composite materials, high-speed steel (HSS), conductive ceramics etc. Its unique feature of using thermal energy to machine electrically conductive materials regardless of hardness is a distinctive advantage in the manufacture of mold, die, automotive, aerospace and surgical components. EDM is a process for eroding and removing material by transient action of electric sparks on electrically conductive materials, one being the workpiece electrode and the other being the tool electrode, immersed in a dielectric fluid and separated by a small gap. The main mode of erosion is caused due to local thermal effect of an electric discharge. The workpiece material is removed by a local high temperature associated with a very high energy density caused by ionisation within the discharge column between the workpiece and electrode. Hence, the material removal mechanism of EDM process is thermal erosion caused by melting and vaporisation. Some of the most important performance measures (responses) of EDM process are material removal rate (MRR), electrode wear rate (EWR) and surface roughness (SR), which are affected by several process parameters, e.g. workpiece polarity, pulse-on-time, duty factor, open discharge voltage, discharge current, materials used as dielectric fluid etc. Improved performance of EDM process can only be achieved by setting the optimal levels for those process parameters.

Taguchi method (Phadke, 1989) of experimental design is a widely used technique for accomplishing the task for optimization of process parameters. The advantage of Taguchi method is that it facilitates assessing the effects of a large number of process parameters using lesser number of experimental trials. Moreover, Taguchi method employs signal-to-noise (SN) ratio with 
simultaneous consideration of achieving the target and reducing variability around the target value of a response variable. However, one limitation of Taguchi method is that it can optimize only one response variable at a time (Park, 2001). Yadav et al., (2008), Patel et al. (2009) and Abdulkareem et al. (2010) determined the optimal process conditions for MRR, SR and EWR respectively using Taguchi method. Secilmis et al. (2009) investigated the influences of EDM process parameters on titaniumporcelain adhesion. Somashekhar et al. (2010) identified the optimal machining condition that would maximize MRR in microEDM, using artificial neural network and genetic algorithm.

On the other hand, Chiang (2007), Bhaduri et al. (2009) and Lin et al. (2009) studied the effects of EDM process parameters on multiple responses. But instead of simultaneous optimization of multiple responses, they determined the optimal process settings for each response separately. Chiang (2007) adopted response surface methodology (RSM) to explain the influences of four machining parameters on MRR, EWR and SR, and found that the optimal parametric conditions for the three responses were different. Bhaduri et al. (2009) studied the effects of four EDM process parameters on four responses, e.g. MRR, EWR, radial overcut and taper angle while machining $\mathrm{TiN}-\mathrm{Al}_{2} \mathrm{O}_{3}$ composite, and also observed that the optimal parametric conditions for the four responses were different. Lin et al. (2009) investigated the effects of six control factors on machining performance of EDM process on conductive ceramics and found that the required optimal settings for the three responses (MRR, EWR and SR) were not the same. However, in reality, the process engineer needs to carry out the machining operation using only one factor level combination. In such situations, use of experience and engineering knowledge are required for resolving the conflict (Phadke, 1989). However, by human judgment, validity of the experimental results cannot be easily assured. Moreover, contradictory results may be reached by different engineers implying that the uncertainty in the optimal factor levels will be increased.

With the aim to alleviate the problems of using experience and engineering knowledge, some other researchers have been motivated to apply different techniques of multi-response optimization for determining the optimal process conditions for EDM processes. Lin et al. (2000) used Taguchi method with fuzzy logic; Lin and Lin (2005) employed grey-fuzzy logic; Kansal et al. (2006) adopted Taguchi method with utility concept; Kanagarajan et al. (2009) used non-linear goal programming based on genetic algorithm; El-Taweel (2009), Pradhan and Biswas (2011), Baraskar et al. (2011) utilized desirability function approach; Jung and Kwon (2010), Natarajan and Arunachalam (2011), Singh (2012) applied grey relational analysis (GRA); and Sanchez et al. (2011) developed an inverse model for simultaneous optimization of multiple responses of EDM processes. But none of these methods take care of the possible correlation between the response variables that may exist, whereas, correlation analysis reveals that some of the responses of EDM process are usually correlated. For example, the correlation coefficient between MRR and EWR in the experimental data of Lin et al. (2009) is found as -0.87 , which is statistically significant at $5 \%$ level. So, ideally, a principal component analysis (PCA)-based method that can take into account the possible correlation between the responses should be used for multi-response optimization of EDM processes. Recently, Chakravorty et al. (2012) analyzed some experimental data on EDM processes using four PCA-based approaches, e.g. PCA-based GRA method, PCA-based proportion of quality loss reduction (PQLR) method, PCA-based technique for order preference by similarity to ideal solution (TOPSIS) method and weighted principal component (WPC) method. The authors found that the PCA-based approaches, in general, would lead to better optimization performance and among the four PCA-based approaches, PCA-based PQLR method would result in the best optimization performance.

In this paper, a modified PCA-based utility theory (UT) approach for optimization of correlated responses is presented and then two reported experimental data on EDM processes are analyzed using the modified procedure and PCA-based PQLR method. The results show that the modified PCA-based UT approach results in better optimization performance than PCA-based PQLR method. This implies that the best performance of EDM process can be achieved by applying the modified PCA-based UT method.

\section{Literature review on multi-response optimization methods}

The goal of multi-response optimization is to find out the settings of the control factors that can achieve an optimal compromise of the response variables. With this aim, several multi-response optimization approaches, mostly RSM-based, have been proposed in the statistical literature. These include desirability function approach (Derringer and Suich, 1980; Kim and Lin, 2000), multivariate loss function approach (Pignatiello, 1993; Tsui, 1999), Mahalanobis distance minimization approach (Khuri and Conlon, 1981) etc. These mathematically rigorous techniques are usually impractical for application by the engineers who may not have a strong background in mathematics/statistics.

Some researchers have been motivated to make use of techniques of artificial intelligence, like artificial neural network (Panda and Mahapatra, 2010), genetic algorithm (Sardinas et al., 2009) etc. for optimization of multi-response processes. In these approaches, the parameters can be set optimally, but nothing can be learned about the relationship between the control factors and responses, and so they do not help the engineers to acquire sufficient experience during the optimization of the concerned process.

Taguchi method has gained enormous popularity among the managers and engineers in recent years. However, one limitation of Taguchi method is that it focuses on optimization of single response only. Considerable researches have been carried out aiming to establish an objective method for solving multi-response optimization problems using Taguchi method. Some of the proposed methods in this regard, usually found in engineering literature, are weighted signal-to-noise (WSN) ratio method (Tai et al, 1992), multi-response signal-to-noise (MRSN) ratio method (Ramakrishnan and Karunamoorthy, 2009), GRA method (Pan et al., 2007) and VIKOR method (Tong et al., 2007). In these approaches, the quality loss or SN ratio of individual responses are first 
converted into an overall process performance index (PPI) and then, the factor-level combination that would optimize the PPI is determined examining the level averages on the PPI. All the necessary computations for this purpose can be performed using Excel worksheet. So these methods can be well acceptable to the practitioners.

However, none of the above methods for multi-response optimization takes into account the possible correlations between the responses that may exist. In order to take care of the possible correlations between the response variables, many researchers (Tong and Wang, 2002; Tong et al., 2005; Liao, 2006; Datta and Mahapatra, 2010) proposed to make use of PCA (Johnson and Wichern, 1996). The PCA technique can transform several related variables into a smaller number of uncorrelated principal components, which are linear combinations of the original variables. The past researchers suggested optimizing the process settings with respect to principal components instead of the original response variables. Tong and Wang (2002) proposed PCA-based GRA method, Wu and Chyu (2004) suggested PCA-based PQLR method, Tong et al. (2005) presented PCA-based TOPSIS method and Liao (2006) proposed weighted principal component (WPC) method. Recently, Datta and Mahapatra (2010) proposed the PCA-based UT approach to optimize multiple responses that may be correlated. The PCA-based UT approach seems to be very appealing. But one apparent problem in it is that it does not make use of Taguchi's SN ratio concept appropriately. It is observed that if Taguchi's SN ratio concept is integrated into the PCA-based UT approach, it can be the most systematic and practical approach for optimization of correlated responses.

\section{Taguchi’s SN ratio concept}

Taguchi's robust design method aims at achieving a target value and minimizing variability around the target value. Taguchi used a quadratic function for modeling quality loss whenever the characteristic deviates from its target value and considered SN ratio as a measure of performance. The most notable aspect of SN ratio is that it combines location and dispersion of a response variable in a single performance measure, whereas, other methods examine mean and variance as separate performance measures. Taguchi (Phadke, 1989) categorized the response variables mainly into three different classes, e.g. smaller-the-better (STB), larger-the-better (LTB) and nominal-the-best (NTB). The formulae for computation of SN ratio $\left(\eta_{i j}\right)$ for $j^{\text {th }}$ response corresponding to $i^{\text {th }}$ trial $(i=1,2, \ldots, m ; j=1,2, \ldots, p)$ are different for different types of response variables, and these are given as follows:

$$
\begin{aligned}
& \text { For STB response variable, } \eta_{i j}=-10 \times \log _{10}\left(\frac{1}{n} \sum_{k=1}^{n} y_{i j k}^{2}\right) \\
& \text { For LTB response variable, } \eta_{i j}=-10 \times \log _{10}\left(\frac{1}{n} \sum_{k=1}^{n} \frac{1}{y_{i j k}^{2}}\right) \\
& \text { For NTB response variable, } \eta_{i j}=10 \times \log _{10}\left(\frac{\bar{y}_{i j}^{2}}{s_{i j}^{2}}\right)
\end{aligned}
$$

where $\bar{y}_{i j}=\frac{1}{n} \sum_{k=1}^{n} y_{i j k}, s_{i j}{ }^{2}=\frac{1}{n-1} \sum_{k=1}^{n}\left(y_{i j k}-\bar{y}_{i j}\right)^{2}, n$ represents the number of repeated experiments and $y_{i j k}$ is the experimental value of $j^{\text {th }}$ response variable in $i^{\text {th }}$ trial at $k^{\text {th }}$ replication. Since $\log$ is a monotone function, minimization of quality loss is equivalent to maximization of SN ratio, and regardless of type of the quality characteristic, a higher SN ratio always implies better quality. As highlighted by Taguchi, the logarithmic transformation improves additivity of effects of two or more control factors (Phadke, 1989), and therefore, optimization of SN ratio is always preferred to quality loss. Another advantage of SN ratio is that it is always expressed in decibel $(\mathrm{dB})$ unit.

\section{Utility concept and modified PCA-based UT approach}

\subsection{Utility concept}

Utility can be defined as the usefulness of a product or process in reference to the expectations of the users. The overall usefulness of a product/process can be represented by a unified index, termed as utility which is the sum of individual utilities of various quality characteristics of the product/process. The methodological basis for utility approach is to transform the estimated value of each quality characteristic into a common index.

If $X_{j}$ is the measure of effectiveness of $j^{\text {th }}$ attribute or quality characteristic (response variable) and there are $p$ attributes evaluating the outcome space, then the joint utility function (Derek, 1982) can be expressed as:

$$
U\left(X_{1}, X_{2}, \ldots, X_{p}\right)=f\left(U_{1}\left(X_{1}\right), U_{2}\left(X_{2}\right), \ldots, U_{p}\left(X_{p}\right)\right)
$$

where $U_{j}\left(X_{j}\right)$ is the utility of $j^{\text {th }}$ attribute or quality characteristic. 
The overall utility function is the sum of individual utilities if the attributes are independent, and is given as follows:

$$
U\left(X_{1}, X_{2}, \ldots, X_{p}\right)=\sum_{j=1}^{p} U_{j}\left(X_{j}\right)
$$

The attributes may be assigned weights depending upon the relative importance or priorities of the characteristics. The overall utility function after assigning weights to the attributes can be expressed as:

$$
U\left(X_{1}, X_{2}, \ldots, X_{p}\right)=\sum_{j=1}^{p} W_{j} U_{j}\left(X_{j}\right)
$$

where $W_{j}$ is the weight assigned to $j^{\text {th }}$ attribute. The sum of the weights for all the attributes must be equal to 1 .

A preference scale for each attribute (response variable) is constructed for determining its utility value. Two arbitrary numerical values (preference numbers) 0 and 9 are assigned to the just acceptable and the best value of the response variable respectively. The preference number $\left(P_{j}\right)$ for $j^{\text {th }}$ response variable can be expressed on a logarithmic scale as follows (Kumar et al., 2000):

$$
P_{j}=A_{j} \times \log \left(\frac{X_{j}}{X_{j}^{\prime}}\right)
$$

where $X_{j}=$ value of $j^{\text {th }}$ response variable, $X_{j}^{\prime}=$ just acceptable value of $j^{\text {th }}$ response variable and $A_{j}=$ constant for $j^{\text {th }}$ response variable. The value of $A_{j}$ can be found by the condition that if $X_{j}=X_{j}^{B}$ (where $X_{j}^{B}$ is the optimal or best value for $j^{\text {th }}$ response), then $P_{j}=9$. Therefore,

$$
A_{j}=\frac{9}{\log \left(\frac{X_{j}^{B}}{X_{j}^{\prime}}\right)}
$$

The overall utility $(U)$ can be calculated as follows:

$$
U=\sum_{j=1}^{p} W_{j} P_{j}
$$

subject to the condition that $\sum_{j=1}^{p} W_{j}=1$.

\subsection{Dutta and Mahapatra (2010) proposed PCA-based UT approach}

Suppose that there are $m$ experimental trials and in each trial, values (single or average) of a set of $p$ performance characteristics (response variables) are observed. Let the observed values of each response variable are transformed/normalized into $[0,1]$ interval in such a way that the most desired value of a response variable is 1 . The normalized experimental results can be expressed in the following series:

$$
\begin{aligned}
& X_{1}, X_{2}, X_{3}, \ldots, X_{i}, \ldots, X_{m} \\
& \text { where } \\
& X_{1}=\left\{X_{11}, X_{12}, \ldots, X_{1 k}, \ldots, X_{1 p}\right\} \\
& X_{i}=\left\{X_{i 1}, X_{i 2}, \ldots, X_{i k}, \ldots, X_{i p}\right\} \\
& X_{m}=\left\{X_{m 1}, X_{m 2}, \ldots, X_{m k}, \ldots, X_{m p}\right\}
\end{aligned}
$$

Here, $X_{i}$ represents the normalized experimental results in $i^{\text {th }}$ trial and is called as a comparative sequence.

Let, $X_{0}=\left\{X_{01}, X_{02}, \ldots, X_{0 k}, \ldots, X_{0 p}\right\}$ be the reference sequence. The values of the elements in the reference sequence are the optimal values (ideal or desired values) of the quality characteristics. With respect to the normalized comparative sequences, all the elements in the reference sequence will be $1 . X_{0}$ and $X_{i}$ both include $p$ elements, and $X_{0 j}$ and $X_{i j}$ represent the numeric value of $j^{t h}(j=1,2, \ldots, p)$ element in the reference sequence and $i^{\text {th }}$ comparative sequence respectively. So the amount of deviations of different response variables from their ideal values can be estimated for different trials. Datta and Mahapatra (2010) called these deviations as quality losses (Taguchi's definition for quality loss is different). The quality losses can be appropriately converted into preference numbers and overall utility values, using Eqns. (7)-(9). The overall utility value is considered as the multi-response process performance index (MPPI). Then, the optimal process setting can be determined by examining the level averages of the control factors on the overall utility value (MPPI). This approach should work well if the response variables are independent.

Datta and Mahapatra (2010) observed that the response variables might not be always independent. Therefore, they proposed to estimate the quality losses with respect to principal component scores (i.e. numeric values of the principal components) of the reference and comparative sequences instead of the normalized values of the response variables, and then to compute the utility values. By applying PCA technique, $p$ related variables can be transformed into $p$ uncorrelated principal components, each of 
which is linear combinations of the original variables. The variability in the original $p$ response variables corresponding to a trial can now be explained by these $p$ uncorrelated principal components. Let $[X]_{\operatorname{mxp}}$ is subjected to PCA, and the resulting eigenvalue and eigenvector of $l^{\text {th }}(l=1,2, \ldots, p)$ principal component are $\lambda_{l}$ and $\beta_{l}$ respectively. Then, the principal component score (PCS) of $l^{\text {th }}$ principal component corresponding to $i^{\text {th }}$ trial or comparative sequence, can be obtained as follows:

$$
P C S_{i l}=a_{l 1} X_{i 1}+a_{l 2} X_{i 2}+\ldots+a_{l p} X_{i p}(i=1,2, \ldots, m \text { and } l=1,2, \ldots, p)
$$

where $a_{l 1}, a_{l 2}, \ldots, a_{l p}$ are the elements of the eigenvector $\beta_{l}$, and $a_{l 1}^{2}+a_{l 2}^{2}+\ldots+a_{l p}^{2}=1$.

Similarly, the principal component scores for the reference sequence can also be estimated. So, quality losses for the principal components in different trials can now be estimated. For example, the quality loss for $l^{\text {th }}$ principal component in $i^{\text {th }}$ trial can be estimated as $Z_{i l}-Z_{0 l}$, where $Z_{i l}$ and $Z_{0 l}$ are the scores for $l^{\text {th }}$ principal component of $i^{\text {th }}$ comparative sequence and reference sequence respectively. The UT can be applied on the computed quality losses to calculate the overall utility value (MPPI).

Based on the above logic, Datta and Mahapatra (2010) proposed the following seven steps for optimizing correlated responses:

Step 1: Transform (normalize) the responses into [0, 1] interval.

Step 2: Perform PCA on the normalized responses and obtain eigenvectors of the principal components.

Step 3: Compute principal component scores for the reference sequence and also for the comparative sequences (trials).

Step 4: $\quad$ Estimate the quality losses in different trials with respect to each principal component.

Step 5: Apply UT for estimating overall utility values corresponding to different trials.

Step 6: Convert the overall utility value of each trial into SN ratio.

Step 7: Select the factor-level combination that maximizes the SN ratio.

\subsection{The Proposed Modified PCA-based UT approach}

It can be noticed that Datta and Mahapatra (2010) computed the overall utility values for different trials considering the observed values of the response variables as the input data and then they converted the overall utility value of each trial into the corresponding SN ratio. Since higher utility value is always desirable, they computed the SN ratio considering utility value as a LTB type characteristic. They selected the optimal factor-level combination examining the level averages on the SN ratio. But, it is found that converting the overall utility value into SN ratio does not add any value to the decision-making process. This is because that the factor-level combination selected by examining the level averages on the overall utility value and the factor-level combination selected by examining the level averages on SN ratio would be always same.

One of the most significant contributions of Taguchi method is the introduction of SN ratio concept as a measure of performance. The most notable aspect of $\mathrm{SN}$ ratio is that it combines location and dispersion of a response variable in a single performance measure. According to Taguchi's philosophy (Phadke, 1989), the aim of process optimization should be to optimize the SN ratio of a response variable instead of the value of the response variable. Otherwise, one may arrive at an optimal solution at which the mean value of a response variable is optimized, but at the same time, variability of the response variable is increased, which is not at all acceptable. Optimization of a process with respect to SN ratio of a response variable prevents arriving at such an unacceptable optimal solution since SN ratio combines the location and dispersion of the response variable together. Keeping these in mind, the following seven steps are proposed for implementing the PCA-based UT approach for optimization of correlated responses.

Step 1: Transform all the observed experimental values of each response variable into SN ratios, using Eqn. (1), (2) or (3), as appropriate.

Step 2: Scale the computed SN ratio values of each response variable into $[0,1]$ interval using the following equation:

$$
Y_{i j}=\frac{\eta_{i j}-\eta_{j}^{\min }}{\eta_{j}^{\max }-\eta_{j}^{\min }}
$$

where $Y_{i j}=$ scaled SN ratio value for $j^{t h}(j=1,2, \ldots, p)$ response variable in $i^{t h}$ trial, $\eta_{j}^{\min }=\min \left\{\eta_{1 j}, \eta_{2 j}, \ldots, \eta_{m j}\right\}$ and $\eta_{j}^{\max }=\max \left\{\eta_{1 j}, \eta_{2 j}, \ldots, \eta_{m j}\right\}$. The purpose of the scaling operation is to reduce the variability among different responses. With respect to the scaled values of the response variables, all the elements in the reference sequence would be 1 ..

Step 3: Perform PCA on the scaled SN ratio values of the response variables to obtain eigenvectors of the uncorrelated principal components. The option of performing PCA is available in any standard statistical software, e.g. STATISTICA, MINITAB and STATGRAPHICS.

Step 4: Compute the values of each principal component (PCS) for different comparative sequences (trials) and also for the reference sequence.

The value of $l^{\text {th }}$ principal component corresponding to $i^{\text {th }}$ comparative sequence, $Z_{i l}$ can be obtained as follows:

$$
Z_{i l}=a_{l 1} Y_{i 1}+a_{l 2} Y_{i 2}+\ldots+a_{l p} Y_{i p} \quad(i=1,2, \ldots, m \text { and } l=1,2, \ldots, p)
$$

On the other hand, all the elements in the reference sequence are 1 and therefore, the value of $l^{\text {th }}$ principal component 
of the reference sequence can be estimated using the following equation:

$$
Z_{0 l}=a_{l 1} \times 1+a_{l 2} \times 1+\ldots+a_{l p} \times 1 \quad(l=1,2, \ldots, p)
$$

Step 5: $\quad$ Estimate the quality losses in different trials with respect to each principal component.

The quality loss for $t^{\text {th }}$ principal component in $i^{\text {th }}$ trial can be considered as the absolute deviation of $Z_{i l}$ value from $Z_{0 l}$ value. Therefore, the quality loss for $l^{\text {th }}$ principal component in $i^{\text {th }}$ trial, $L_{i l}$ can be estimated using the following equation:

$$
L_{i l}=\left|Z_{i l}-Z_{0 l}\right|
$$

Step 6: Apply UT for estimating the overall utility values for different trials.

Using Eqns. (7) and (8), the estimated quality losses for different principal components can be appropriately converted to preference numbers. Then, the overall utility values for different trials can be estimated using Eqn. (9). It is suggested here to consider the proportion of variation expressed by different principal components as their weights.

Step 7: Select the factor-level combination that maximizes the overall utility value.

\section{PCA-based PQLR method (Wu and Chyu, 2004)}

For any process, there is a starting condition. Let, the $\mathrm{SN}$ ratio for a response variable at the starting condition is $\eta_{o}$ and under a new process condition, the $\mathrm{SN}$ ratio is $\eta^{\prime}$. This implies that quality loss $(L)$ at the starting condition and quality loss $\left(L^{\prime}\right)$ at the new condition are $10^{-\left(\eta_{o} / 10\right)}$ and $10^{-\left(\eta^{\prime} / 10\right)}$ respectively. So, the proportion of quality loss reduction $(P Q L R)$ in the new condition is as follows:

$$
P Q L R=10^{-\left(\eta^{\prime}-\eta_{o}\right) / 10}
$$

In Taguchi's experimental design, the factor-level combination for each trial represents a new process condition and so the PQLR value for each response variable corresponding to each trial can be easily estimated. If the responses are correlated, the estimated PQLR values for the responses would also be correlated. Therefore, Wu and Chyu (2004) proposed the PCA-based PQLR method for optimizing the correlated responses. The MPPI in PCA-based PQLR method is simply called as the weighted score $(W S)$.

Determination of the optimal process condition using PCA-based PQLR method includes the following eight steps:

Step 1: Compute the SN ratio of each response variable corresponding to each trial.

Step 2: Calculate the main effect (in terms of SN ratio) of each control factor for each response variable using arithmetic average..

Step 3: Estimate the expected SN ratio at the starting condition (or any arbitrary condition) for each response variable using additive model.

Step 4: Transform the computed SN ratio of each response variable corresponding to each trial into PQLR value using Eqn. (15)

Step 5: $\quad$ Normalize the PQLR values of each response variable. The normalized PQLR value for $j^{\text {th }}$ response variable in $i^{\text {th }}$ trial $\left(Y_{i j}\right)$ can be obtained by replacing $\eta$ by PQLR in the right hand side of Eqn. (11).

Step 6: Perform PCA on the normalized PQLR values and obtain the values of the principal components.

The value of $l^{\text {th }}(l=1,2, \ldots, p)$ principal component corresponding to $i^{\text {th }}$ trial, $\left(Z_{i l}\right)$ can be obtained using Eqn. (12).

Step 7: Take the absolute value of $Z_{i l}$ and then compute the weighted score (WS) for each trial as follows:

$$
W S_{i}=\sum_{l=1}^{p} w_{l}\left|Z_{i l}\right|
$$

Step 8: Use geometric average to calculate the factor effects based on WS values and then decide the optimal factor-level combination by smaller-the-better factor effects.

\section{Analysis and results}

For illustrating the application of the proposed modified PCA-based UT approach and validating its optimization performance, two sets of the past experimental data on EDM processes are analyzed using the modified PCA-based UT approach as well as PCA-based PQLR method as two separate case studies.

\subsection{Case study 1}

Lin et al. (2000) carried out an experimental study on an EDM machine (KT-200) with the aim to determine the optimal levels of six machining parameters (control factors), e.g. workpiece polarity (A), pulse-on-time (B), duty factor (C), open discharge voltage 
(D), discharge current (E) and dielectric fluid (F) with respect to two performance characteristics (responses) of EDM process. In that study, cylindrical pure copper was used as the electrode and SKD11 was used as the workpiece. The considered two responses were EWR (\%) and MRR (gm/min). While EWR is a STB type response variable, MRR is a LTB type response variable. The experimental layout was designed considering two levels for control factor A and three levels for all other control factors. So, the standard $L_{18}$ orthogonal array was used for the experiments. The experimental data of Lin et al. (2000) are analyzed here using the modified PCA-based UT method and PCA-based PQLR method as case study 1.

The experimental layout along with the computed SN ratios for the two response variables are given in Table 1. It may be noted that the modified PCA-based UT method requires PCA of the scaled SN ratio values (see section 4.3) and PCA-based PQLR method requires PCA of the normalized PQLR values (see section 5). For application of PCA-based PQLR method, at first, the PQLR values corresponding to different trials are estimated for all the responses assuming that $A_{2} B_{2} C_{2} D_{2} E_{2} F_{2}$ is the starting condition and then PQLR values for all the responses are normalized. On the other hand, the SN ratios of the response variables are normalized for application of the modified PCA-based UT method. The normalized SN ratios and normalized PQLR values of the response variables are then subjected to PCA in STATISTICA software. The eigenvalues and the corresponding eigenvectors, obtained from PCA of the scaled SN ratios are given in Table 2. The eigenvalues and the corresponding eigenvectors, derived from PCA of the normalized PQLR values, are shown in Table 3.

Following the remaining steps mentioned in sections 4.3 and 5, values of MPPIs for the modified PCA-based UT method and PCA-based PQLR method, i.e. overall utility and WS values are computed. The MPPI values corresponding to different trials obtained by using the two PCA-based methods are shown in Table 4.

Table 1. Experimental layout and SN ratios of the responses for case study 1

\begin{tabular}{|c|c|c|c|c|c|c|c|c|}
\hline \multirow{2}{*}{$\begin{array}{c}\text { Exp. } \\
\text { No. }\end{array}$} & \multicolumn{7}{|c|}{ Factor assignment } & \multicolumn{2}{c|}{ SN ratio $(\eta)$ in dB } \\
\cline { 2 - 9 } & A & B & C & D & E & F & EWR & MRR \\
\hline 1 & 1 & 1 & 1 & 1 & 1 & 1 & -30.53 & -54.80 \\
\hline 2 & 1 & 1 & 2 & 2 & 2 & 2 & -29.51 & -55.97 \\
\hline 3 & 1 & 1 & 3 & 3 & 3 & 3 & -33.46 & -61.01 \\
\hline 4 & 1 & 2 & 1 & 1 & 2 & 2 & -16.52 & -46.67 \\
\hline 5 & 1 & 2 & 2 & 2 & 3 & 3 & -21.44 & -60.35 \\
\hline 6 & 1 & 2 & 3 & 3 & 1 & 1 & -32.85 & -57.86 \\
\hline 7 & 1 & 3 & 1 & 2 & 1 & 3 & -32.26 & -72.40 \\
\hline 8 & 1 & 3 & 2 & 3 & 2 & 1 & -13.82 & -50.37 \\
\hline 9 & 1 & 3 & 3 & 1 & 3 & 2 & 3.10 & -49.63 \\
\hline 10 & 2 & 1 & 1 & 3 & 3 & 2 & -27.16 & -72.40 \\
\hline 11 & 2 & 1 & 2 & 1 & 1 & 3 & -30.13 & -73.56 \\
\hline 12 & 2 & 1 & 3 & 2 & 2 & 1 & -29.31 & -71.70 \\
\hline 13 & 2 & 2 & 1 & 2 & 3 & 1 & -23.81 & -71.70 \\
\hline 14 & 2 & 2 & 2 & 3 & 1 & 2 & -36.55 & -70.75 \\
\hline 15 & 2 & 2 & 3 & 1 & 2 & 3 & -29.31 & -69.63 \\
\hline 16 & 2 & 3 & 1 & 3 & 2 & 3 & -31.66 & -65.51 \\
\hline 17 & 2 & 3 & 2 & 1 & 3 & 1 & -27.64 & -69.12 \\
\hline 18 & 2 & 3 & 3 & 2 & 1 & 2 & -41.67 & -70.17 \\
\hline
\end{tabular}

Table 2. Eigenvalues and eigenvectors for normalized SN ratios (case study 1)

\begin{tabular}{|c|c|c|}
\hline Principal component & Eigenvalue & Eigenvector \\
\hline First & 1.619 & {$[0.707,0.707]$} \\
\hline Second & 0.381 & {$[0.707,-0.707]$} \\
\hline
\end{tabular}

Table 3. Eigenvalues and eigenvectors for normalized PQLR values (case study 1)

\begin{tabular}{|c|c|c|}
\hline Principal component & Eigenvalue & Eigenvector \\
\hline First & 1.143 & {$[0.707,0.707]$} \\
\hline Second & 0.857 & {$[0.707,-0.707]$} \\
\hline
\end{tabular}

Table 5 gives the level averages of the control factors on the overall utility and WS values. In this table, the desired level averages are bold faced. Larger value of overall utility signifies better quality. Consequently, the optimal conditions for factors A, $\mathrm{B}, \mathrm{C}, \mathrm{D}, \mathrm{E}$ and $\mathrm{F}$ with respect to overall utility value can be set as $\mathrm{A}_{1} \mathrm{~B}_{3} \mathrm{C}_{3} \mathrm{D}_{1} \mathrm{E}_{3} \mathrm{~F}_{2}$, i.e. workpiece polarity = negative, pulse-on time $=300 \mu \mathrm{s}$, duty factor $=0.7$, open discharge voltage $=100 \mathrm{~V}$, discharge current $=6 \mathrm{~A}$ and dielectric fluid $=\mathrm{Kerosene}+\mathrm{Al}_{2} \mathrm{O}_{3}$ powder. On the other hand, as smaller value of WS implies better quality, the optimal condition with respect to WS value can be 
chosen as $\mathrm{A}_{1} \mathrm{~B}_{3} \mathrm{C}_{2} \mathrm{D}_{1} \mathrm{E}_{2} \mathrm{~F}_{2}$, i.e. workpiece polarity = negative, pulse-on time $=300 \mu \mathrm{s}$, duty factor $=0.5$, open discharge voltage $=100 \mathrm{~V}$, discharge current $=4 \mathrm{~A}$ and dielectric fluid $=$ Kerosene $+\mathrm{Al}_{2} \mathrm{O}_{3}$ powder. It may be noted that the optimal process conditions obtained by the application of the modified PCA-based UT method and PCA-based PQLR method are different.

Table 4. MPPI values for the experimental data of case study 1

\begin{tabular}{|c|c|c|}
\hline \multirow{2}{*}{$\begin{array}{c}\text { Exp. } \\
\text { No. }\end{array}$} & \multicolumn{2}{|c|}{ Multi-response process performance index (MPPI) } \\
\hline 1 & Overall utility & Weighted score (WS) \\
\hline 2 & 1.480 & 0.055 \\
\hline 3 & 1.502 & 0.045 \\
\hline 4 & 1.051 & 0.112 \\
\hline 5 & 3.745 & 0.002 \\
\hline 6 & 2.577 & 0.033 \\
\hline 7 & 1.172 & 0.095 \\
\hline 8 & 0.628 & 0.553 \\
\hline 9 & 3.599 & 0.002 \\
\hline 10 & 7.920 & 0.001 \\
\hline 11 & 0.566 & 0.545 \\
\hline 12 & 0.439 & 0.714 \\
\hline 13 & 0.670 & 0.467 \\
\hline 14 & 0.657 & 0.462 \\
\hline 15 & 1.847 & 0.401 \\
\hline 16 & 1.003 & 0.291 \\
\hline 17 & 1.418 & 0.120 \\
\hline 18 & 1.037 & 0.258 \\
\hline & 0.576 & 0.754 \\
\hline
\end{tabular}

Table 5 Level averages on overall utility and WS values for case study 1

\begin{tabular}{|c|c|c|c|c|c|c|}
\hline \multirow{2}{*}{ Factor } & \multicolumn{3}{|c|}{ Overall utility } & \multicolumn{3}{c|}{ Weighted score (WS) } \\
\cline { 2 - 7 } & Level 1 & Level 2 & Level 3 & Level 1 & Level 2 & Level 3 \\
\hline A & $\mathbf{2 . 6 3 1}$ & 0.913 & - & $\mathbf{0 . 0 2 4}$ & 0.394 & - \\
\hline B & 0.951 & 1.834 & $\mathbf{2 . 5 3 0}$ & 0.192 & 0.085 & $\mathbf{0 . 0 5 8}$ \\
\hline C & 1.416 & 1.833 & $\mathbf{2 . 0 6 5}$ & 0.112 & $\mathbf{0 . 0 7 8}$ & 0.108 \\
\hline D & $\mathbf{2 . 6 0 4}$ & 1.102 & 1.609 & $\mathbf{0 . 0 4 6}$ & 0.226 & 0.092 \\
\hline E & 1.024 & 1.990 & $\mathbf{2 . 3 0 2}$ & 0.293 & $\mathbf{0 . 0 3 9}$ & 0.084 \\
\hline F & 1.436 & $\mathbf{2 . 6 9 3}$ & 1.186 & 0.092 & $\mathbf{0 . 0 5 3}$ & 0.193 \\
\hline
\end{tabular}

It is important to notice that the overall utility and WS values are essentially used as the tools for multi-response optimization. The quality of performance of a process can actually be judged by the expected SN ratio values of the individual response variables at the derived optimal parametric condition. Therefore, the average SN ratio values of the response variables at different levels of the control factors are computed (based on the values given in Table 1) and then, the expected SN ratio values for the response variables at the two optimal solutions, derived by using the two PCA-based approaches, are estimated employing the additive model (Phadke, 1989). The expected SN ratio values for each response variable under the two different sets of optimal conditions are displayed in Table 6 . The results in Table 6 reveal that by increasing duty factor from 0.5 to 0.7 and discharge current from 4A to 6A, the SN ratio value for EWR increases by $0.74 \mathrm{~dB}$ (the value of EWR reduce from 2.506 to 2.301 ) but the $\mathrm{SN}$ ratio value for MRR deceases by $0.02 \mathrm{~dB}$ (the value of MRR reduce from 0.002941 to 0.002934$)$ only. In effect, the total SN ratio under the optimal condition derived by using the modified PCA-based UT approach becomes considerably higher (1.23\%) than the total SN ratio under the optimal condition that is determined by the PCA-based PQLR method. This result is indicative that the modified PCA-based UT approach can give better optimisation performance. 
Table 6. Predicted SN ratios for the response variables (case study 1)

\begin{tabular}{|c|c|c|c|c|}
\hline \multirow{2}{*}{ Optimization method } & Optimal condition & \multicolumn{2}{|c|}{ Expected SN ratio } & \multirow{2}{*}{$\begin{array}{c}\text { Total SN } \\
\text { ratio }\end{array}$} \\
\cline { 3 - 4 } & EWR & MRR & \\
\hline $\begin{array}{c}\text { Modified PCA-based UT } \\
\text { approach }\end{array}$ & $\mathrm{A}_{1} \mathrm{~B}_{3} \mathrm{C}_{3} \mathrm{D}_{1} \mathrm{E}_{3} \mathrm{~F}_{2}$ & $-7.24 \mathrm{~dB}$ & $-50.65 \mathrm{~dB}$ & $-57.89 \mathrm{~dB}$ \\
\hline PCA-based PQLR method & $\mathrm{A}_{1} \mathrm{~B}_{3} \mathrm{C}_{2} \mathrm{D}_{1} \mathrm{E}_{2} \mathrm{~F}_{2}$ & $-7.98 \mathrm{~dB}$ & $-50.63 \mathrm{~dB}$ & $-58.61 \mathrm{~dB}$ \\
\hline
\end{tabular}

\subsection{Case study 2}

Kansal et al. (2006) investigated the optimization performance of EDM process when silicon powder was suspended into the dielectric fluid used in EDM. In that study, the impacts of four process parameters, i.e. concentration of silicon powder added into dielectric fluid (A), peak current (B), pulse duration (C) and duty cycle (D) were studied with consideration of multiple characteristics, including MRR $\left(\mathrm{mm}^{3} / \mathrm{min}\right)$, SR $(\mu \mathrm{m})$ and TWR $\left(\mathrm{mm}^{3} / \mathrm{min}\right)$. While MRR is a LTB type response variable, SR and TWR are STB type response variables. Three levels for all the process parameters were selected. So the experimental layout was prepared using $L_{9}$ orthogonal array.

The experimental layout and the computed SN ratio values for the three responses are given in Table 7. For application of PCAbased PQLR method, the PQLR values corresponding to different trials are estimated for all the responses assuming that $\mathrm{A}_{2} \mathrm{~B}_{2} \mathrm{C}_{2} \mathrm{D}_{2}$ is the starting condition. The SN ratio and PQLR values are normalized and then, subjected to PCA separately using STATISTICA software. The eigenvalues and the corresponding eigenvectors for the scaled SN ratios are given in Table 8. Table 9 shows the eigenvalues and the corresponding eigenvectors for the normalized PQLR values.

Now, the MPPI values for the modified PCA-based UT method and PCA-based PQLR method are computed. The MPPI values corresponding to different trials obtained employing the two PCA-based methods are shown in Table 10.

Table 7. Experimental layout and computed SN ratios (case study 2)

\begin{tabular}{|c|c|c|c|c|c|c|c|}
\hline \multirow{2}{*}{$\begin{array}{c}\text { Exp. } \\
\text { No. }\end{array}$} & \multicolumn{3}{|c|}{ Factor assignment } & \multicolumn{3}{c|}{ SN ratio $(\eta)$ in dB } \\
\cline { 2 - 8 } & A & B & C & D & MRR & SR & TWR \\
\hline 1 & 1 & 1 & 1 & 1 & -5.02 & -1.39 & 11.70 \\
\hline 2 & 1 & 2 & 2 & 2 & -3.08 & -2.83 & 9.68 \\
\hline 3 & 1 & 3 & 3 & 3 & -1.41 & -3.58 & 5.67 \\
\hline 4 & 2 & 1 & 2 & 3 & -2.33 & -1.26 & 11.70 \\
\hline 5 & 2 & 2 & 3 & 1 & -1.66 & -2.90 & 9.68 \\
\hline 6 & 2 & 3 & 1 & 2 & 0.78 & -3.60 & 7.34 \\
\hline 7 & 3 & 1 & 3 & 2 & -1.73 & -0.44 & 11.87 \\
\hline 8 & 3 & 2 & 1 & 3 & -0.68 & -2.02 & 11.70 \\
\hline 9 & 3 & 3 & 2 & 1 & 1.65 & -2.19 & 8.94 \\
\hline
\end{tabular}

Table 8. Eigenvalues and eigenvectors for normalized SN ratios (case study 2)

\begin{tabular}{|c|c|c|}
\hline Principal component & Eigenvalue & Eigenvector \\
\hline First & 2.15 & {$[0.443,-0.624,-0.644]$} \\
\hline Second & 0.73 & {$[0.891,0.383,0.243]$} \\
\hline Third & 0.13 & {$[-0.095,0.682,-0.725]$} \\
\hline
\end{tabular}

Table 9. Eigenvalues and eigenvectors for normalized PQLR values (case study 2)

\begin{tabular}{|c|c|c|}
\hline Principal component & Eigenvalue & Eigenvector \\
\hline First & 1.92 & {$[0.246,0.682,0.689]$} \\
\hline Second & 0.94 & {$[0.986,-0.207,-0.141]$} \\
\hline Third & 0.14 & {$[-0.046,-0.702,0.711]$} \\
\hline
\end{tabular}


Table 10. MPPI values for experimental data of case study 2

\begin{tabular}{|c|c|c|}
\hline \multirow{2}{*}{$\begin{array}{c}\text { Exp. } \\
\text { No. }\end{array}$} & Multi-response process performance index (MPPI) \\
\cline { 2 - 3 } & Overall utility & Weighted score (WS) \\
\hline 1 & 4.062 & 0.560 \\
\hline 2 & 2.708 & 0.603 \\
\hline 3 & 0.212 & 0.942 \\
\hline 4 & 7.546 & 0.274 \\
\hline 5 & 2.419 & 0.509 \\
\hline 6 & 1.000 & 0.787 \\
\hline 7 & 5.864 & 0.150 \\
\hline 8 & 5.937 & 0.261 \\
\hline 9 & 3.808 & 0.386 \\
\hline
\end{tabular}

Table 11 gives the level averages of the control factors on overall utility and WS values. Here, the optimal conditions for factors $\mathrm{A}, \mathrm{B}, \mathrm{C}$ and $\mathrm{D}$ with respect to overall utility value and $\mathrm{WS}$ value are chosen as $\mathrm{A}_{3} \mathrm{~B}_{1} \mathrm{C}_{2} \mathrm{D}_{3}$ (i.e. powder concentration $=2 \mathrm{~g} / \mathrm{L}$, peak current $=3 \mathrm{~A}$, pulse duration $=100 \mu \mathrm{s}$ and duty cycle $=0.9$ ) and $\mathrm{A}_{3} \mathrm{~B}_{1} \mathrm{C}_{3} \mathrm{D}_{3}$ (i.e. powder concentration $=2 \mathrm{~g} / \mathrm{L}$, peak current $=3 \mathrm{~A}$, pulse duration $=150 \mu$ s and duty cycle $=0.9$ ) respectively.

Table 11. Level averages on overall utility and WS values for case study 2

\begin{tabular}{|c|c|c|c|c|c|c|}
\hline \multirow{2}{*}{ Factor } & \multicolumn{3}{|c|}{ Overall utility } & \multicolumn{3}{c|}{ Weighted score (WS) } \\
\cline { 2 - 7 } & Level 1 & Level 2 & Level 3 & Level 1 & Level 2 & Level 3 \\
\hline A & 2.327 & 3.655 & $\mathbf{5 . 2 0 3}$ & 0.683 & 0.479 & $\mathbf{0 . 2 4 7}$ \\
\hline B & $\mathbf{5 . 8 2 4}$ & 3.688 & 1.674 & $\mathbf{0 . 2 8 4}$ & 0.431 & 0.659 \\
\hline C & 3.666 & $\mathbf{4 . 6 8 7}$ & 2.832 & 0.536 & 0.421 & $\mathbf{0 . 4 1 6}$ \\
\hline D & 3.430 & 3.191 & $\mathbf{4 . 5 6 5}$ & 0.479 & 0.414 & $\mathbf{0 . 4 0 7}$ \\
\hline
\end{tabular}

The average SN ratio values of the responses at different levels of the control factors are computed (based on the values given in Table 7) and then, the expected SN ratio values for the response variables at the two optimal solutions are estimated employing the additive model (Phadke, 1989). The expected SN ratio values for each response variable under the two different sets of optimal conditions are displayed in Table 12. The results in Table 12 reveal that by changing the pulse duration from $150 \mu \mathrm{s}$ to $100 \mu \mathrm{s}$ the SN ratio values for MRR, SR and TWR can be increased by $0.34 \mathrm{~dB}$ (the value of MRR increases from 0.807 to 0.839 ), 0.22 $\mathrm{dB}$ (the value of SR decreases from 1.052 to 1.026 ) and $1.04 \mathrm{~dB}$ (the value of TWR decreases from 0.253 to 0.225 ) respectively. In effect, the total SN ratio under the optimal condition derived by using the modified PCA-based UT approach becomes substantially higher $(16.6 \%)$ than the total SN ratio under the optimal condition that is determined by the PCA-based PQLR method. This is indicative that the modified PCA-based UT approach can give better optimisation performance.

Table 12. Predicted SN ratios for the response variables (case study 2)

\begin{tabular}{|l|c|c|c|c|c|}
\hline \multirow{2}{*}{ Optimization method } & \multirow{2}{*}{ Optimal condition } & \multicolumn{3}{c|}{ Expected SN ratio } & \multirow{2}{*}{$\begin{array}{c}\text { Total SN } \\
\text { ratio }\end{array}$} \\
\cline { 3 - 5 } & & MRR & SR & TWR & \\
\hline Modified PCA-based UT approach & $\mathrm{A}_{3} \mathrm{~B}_{1} \mathrm{C}_{2} \mathrm{D}_{3}$ & $-1.52 \mathrm{~dB}$ & $-0.22 \mathrm{~dB}$ & $12.97 \mathrm{~dB}$ & $11.23 \mathrm{~dB}$ \\
\hline PCA-based PQLR method & $\mathrm{A}_{3} \mathrm{~B}_{1} \mathrm{C}_{3} \mathrm{D}_{3}$ & $-1.86 \mathrm{~dB}$ & $-0.44 \mathrm{~dB}$ & $11.93 \mathrm{~dB}$ & $9.63 \mathrm{~dB}$ \\
\hline
\end{tabular}

The expected optimization performance under the optimal process conditions derived by the modified PCA-based UT approach are found to be better in both case studies. Ideally, confirmatory experimental trials using all the optimal factor-level combination derived based on different methods should be carried out for each process and the results of the confirmatory trials need to be compared with the predicted results to validate the model assumptions. However, the current research work is carried out using published experimental data in literature and therefore, there is no scope to carry out the confirmatory trial with the optimal factor level combination for any process. According to Phadke (1989) and Montgomery (2001), the additive model for prediction is usually appropriate. Based on the predicted values, therefore, it may be concluded that the modified PCA-based UT approach results in better optimisation performance.

\section{Conclusions}

Correlation analysis reveals that the multiple performance characteristics of EDM process are usually correlated. The results of past researches indicate that PCA-based PQLR method may be the most appropriate approach for optimization of correlated 
responses of EDM processes. In this paper, a modified PCA-based UT approach for optimizing correlated responses is presented, in which Taguchi's SN ratio concept (one of the most significant contributions of Taguchi) is properly integrated. Two sets of past experimental data on EDM processes are analyzed using the modified PCA-based UT method and PCA-based PQLR method. The comparison of the optimization performances at the optimal conditions derived by the two methods indicates that the optimal condition derived by the modified PCA-based UT method leads to better optimization performance. This implies that the modified PCA-based UT approach can be a promising method for optimizing correlated responses of EDM process.

Acknowledgement: The authors would like to thank the referees for their valuable comments and suggestions which have substantially improved the content and presentation of this paper.

\section{References}

Abdulkreem, S., Khan, A.A. and Konneh, M. 2010. Cooling effect on electrode and process parameters in EDM. Materials and Manufacturing Processes, Vol. 25, pp. 462-466.

Baraskar, S.S., Banwait, S.S. and Laroiya, S.C. 2011. Multi-objective optimisation of electrical discharge machining process using Derringer's desirability function approach. International Journal of Materials Engineering Innovation, Vol. 2, pp. 203-221.

Bhaduri, D., Kuar, A.S., Sarkar, S., Biswas, S.K. and Mitra, S. 2009. Electro discharge machining of titanium nitride-aluminium oxide composite for optimum process criteria yield. Materials and Manufacturing Processes, Vol. 24, pp. 1312-1320.

Chakravorty, R., Gauri, S.K. and Chakraborty, S. 2012. Optimization of correlated responses of EDM process. Materials and Manufacturing Processes, Vol. 27, pp. 337-347.

Chiang, K-T. 2007. Modeling and analysis of the effects of machining parameters on the performance characteristics in the EDM process of $\mathrm{Al}_{2} \mathrm{O}_{3}+\mathrm{TiC}$ mixed ceramic. International Journal of Advanced Manufacturing Technology, Vol. 37, pp. 523-533.

Datta, S. and Mahapatra, S.S. 2010. Simultaneous optimisation of correlated multiple surface quality characteristics of mild steel turned product. Intelligent Information Management, Vol. 2, pp. 26-39.

Derringer, G. and Suich, R. 1980. Simultaneous optimisation of several response variables. Journal of Quality Technology, Vol. 12, pp. 214-219.

Derek, W.B. 1982. Analysis of Optimal Decisions. John Wiley and Sons, New York.

El-Taweel, T.A. 2009. Multi-response optimization of EDM with Al-Cu-Si-TiC P/M composite electrode. International Journal of Advanced Manufacturing Technology, Vol. 44, pp. 100-113.

Johnson, R.A. and Wichern, D.W. 1996. Applied Multivariate Statistical Analysis. Prentice Hall of India Pvt. Ltd., New Delhi.

Jung, J.H. and Kwon, W.T. 2010. Optimisation of EDM process for multiple performance characteristics using Taguchi method and grey relational analysis. Journal of Mechanical Science and Technology, Vol. 24, pp. 1083-1090.

Kanagarajan, D., Karthikeyan, R., Palanikumar, K. and Davim, J.P. 2009. Application of goal programming technique for electro discharge machining (EDM) characteristics of cemented carbide (WC/Co). International Journal of Materials and Product Technology, Vol. 35, pp. 216-227.

Kansal H.K., Singh S., Kumar P. 2006. Performance parameters optimization (multi-characteristics) of powder mixed electric discharge machining (PMEDM) through Taguchi's method and utility concept. Indian Journal of Engineering and Materials Sciences, Vol. 13, pp. 209-216.

Khuri, A.I. and Conlon, M. 1981. Simultaneous optimisation of multiple responses represented by polynomial regression functions. Technometrics, Vol. 23, pp. 363-375.

Kim, K., Lin, D. 2000. Simultaneous optimisation of multiple responses by maximising exponential desirability functions. Journal of Royal Statistical Society-Applied Statistics, Vol. 43, pp. 311-325.

Kumar, P., Barua, P.B. and Gaindhar, J.L. 2000. Quality optimisation (multi-characteristics) through Taguchi technique and utility concept. Quality and Reliability Engineering International, Vol. 16, pp. 475-485.

Liao, H.C. 2006. Multi-response optimisation using weighted principal component. International Journal of Advanced Manufacturing Technology, Vol. 27, pp. 720-725.

Lin, J.L., Wang, K.S., Yan, B.H. and Tarng, Y.S. 2000. Optimisation of the electrical discharge machining process based on the Taguchi method with fuzzy logics. Journal of Materials Processing Technology, Vol. 102, pp. 48-55.

Lin, J.L. and Lin, C.L. 2005. The use of grey-fuzzy logic for the optimisation of the manufacturing process. Journal of Materials Processing Technology, Vol. 160, pp. 9-14.

Lin, Y-C., Wang, A-C. Wang, D-A. and Chen, C-C. 2009. Machining performance and optimising machining parameters of $\mathrm{Al}_{2} \mathrm{O}_{3}$-TiC ceramics using EDM based on the Taguchi method. Materials and Manufacturing Processes, Vol. 24, pp. 667-674.

Montgomery, D.C. 2001. Design and analysis of experiments, $5^{\text {th }}$ edn., Wiley, Singapore.

Natarajan, N. and Arunachalam, R.M. 2011. Experimental investigations and optimisation of process parameters in micro-EDM with multiple performance characteristics. International Journal of Experimental Design and Process Optimisation, Vol. 2, pp. 336-356.

Pan, L.K., Wang, C.C., Wei, S.L. and Sher, H.F. 2007. Optimising multiple quality characteristics via Taguchi method-based grey analysis, Journal of Materials Processing Technology, Vol. 182, pp. 107-116. 
Park, J.M. 2001. On stochastic optimization: Taguchi Methods ${ }^{\mathrm{TM}}$ demystified; its limitations and fallacy clarified. Probabilistic Engineering Mechanics, Vol. 16, pp. 87-101.

Panda, S.S. and Mahapatra, S.S. 2010. Online multi-response assessment using Taguchi and artificial neural network. International Journal of Manufacturing Research, Vol. 5, pp. 305-326.

Patel, K.M., Pandey, P.M. and Rao, P.V. 2009. Determination of an optimum parametric combination using a surface roughness prediction model for EDM of $\mathrm{Al}_{2} \mathrm{O}_{3} / \mathrm{SiCw} / \mathrm{TiC}$ ceramic composite. Materials and Manufacturing Processes, Vol. 24, pp. 675682.

Pignatiello, Jr., J.J. 1993. Strategies for robust multi-response quality engineering. IIE Transactions, Vol. 25, pp. 5-15.

Phadke, M.S. 1989. Quality Engineering Using Robust Design, Prentice Hall, Englewood Cliffs, New Jersey.

Pradhan, M.K. and Biswas, C.K. 2011. Multi-response optimisation of EDM of ALSI D2 tool steel using response surface methodology. International Journal of Machining and Machinability of Materials, Vol. 9, pp. 66-85.

Ramakrishnan, R. and Karunamoorthy, L. 2009. Performance studies of wire electrical discharge machining (WEDM) of Inconel 718. International Journal of Materials and Product Technology, Vol. 35, pp. 119-215.

Sanchez, H.T., Estrems, M. and Faura, F. 2011. Development of an inversion model for establishing EDM input parameters to satisfy material removal rate, electrode wear ratio and surface roughness, International Journal of Advanced Manufacturing Technology, Vol. 57, pp. 189-201.

Sardinas, R.Q., Mengana, J.F. and Davim, J.P. 2009. Multi-objective optimisation of multipass turning by using a genetic algorithm. International Journal of Materials and Product Technology, Vol. 35, pp. 134-144.

Secilmis, A. Olmez, A.M., Dilmec, M., Halkaci, H.S. and Inan, O. 2009. Determination of optimal EDM machining parameters for machined pure titanium-porcelain adhesion. International Journal of Advanced Manufacturing Technology, Vol. 45, pp. 55-61.

Singh, S. 2012. Optimization of machining characteristics in electric discharge machining of $6061 \mathrm{Al} / \mathrm{Al}_{2} \mathrm{O}_{3} \mathrm{p} / 20 \mathrm{P}$ composites by grey relational analysis, International Journal of Advanced Manufacturing Technology, DOI: 10.1007/s00170-012-3984-8.

Somashekhar, K.P; Ramchandran, N.; Mathew, J. 2010. Optimization of material removal rate in micro-EDM using artificial neural network and genetic algorithms. Materials and Manufacturing Processes, Vol. 25, pp. 467-475.

Tai, C.Y., Chen, T.S. and Wu, M.C. 1992. An enhanced Taguchi method for optimising SMT processes. Journal of Electronics Manufacturing, Vol. 2, pp. 91-100.

Tong, L-I. and Wang, C-H. 2002. Multi-response optimisation using principal component analysis and grey relational analysis. International Journal of Industrial Engineering, Vol. 9, pp. 343-350.

Tong, L-I, Wang, C-H. and Chen, H-C. 2005. Optimisation of multiple responses using principal component analysis and technique for order preference by similarity to ideal solution. International Journal of Advanced Manufacturing Technology, Vol. 27, pp. 407-414.

Tong, L.I., Chen, C.C. and Wang, C.H. 2007. Optimisation of multi-response processes using the VIKOR method. International Journal of Advanced Manufacturing Technology, Vol. 31, pp. 1049-1057.

Tsui, K. 1999. Robust design optimisation for multiple characteristic problems. International Journal of Production Research, Vol. 37, pp. 433-445.

Wu, F-C. and Chyu, C-C. 2004. Optimization of correlated multiple quality characteristics robust design using principal component analysis. Journal of Manufacturing System, Vol. 23, pp. 134-143.

Yadav, S.K.S., Yadava, V. and Narayana, V.L. 2008. Experimental study and parameter design of electro-discharge diamond grinding. International Journal of Advanced Manufacturing Technology, Vol. 36, pp. 34-42.

\section{Biographical notes}

Rina Chakravorty is a Faculty Member in the Statistical Quality Control and Operations Research Unit of the Indian Statistical Institute (ISI), New Delhi Centre, India. She received M. Stat. and M. Tech. (Quality, Reliability and Operations Research) degree from the ISI. She is engaged in teaching, research and consultancy works.

Dr. Susanta Kumar Gauri is a Faculty Member in the Statistical Quality Control and Operations Research Unit of the Indian Statistical Institute (ISI), Kolkata, India. He received his Ph.D. (Engineering) degree from Jadavpur University, India. His fields of interest are quality engineering, process optimisation and statistical quality control. He has published several research papers in various international journals.

Dr. Shankar Chakraborty is a Faculty Member in the Production Engineering Department of Jadavpur University, India. He received his Ph.D. (Engineering) degree from Jadavpur University. His fields of interest are quality control, multi-criteria decision making and process optimization. He has published several research papers in various international journals.

Received January 2012

Accepted March 2012

Final acceptance in revised form May 2012 